\title{
LINC00152 promotes proliferation in hepatocellular carcinoma by targeting EpCAM via the mTOR signaling pathway
}

\author{
Jie Ji ${ }^{1, *}$, Junwei Tang ${ }^{1, *}$, Lei Deng ${ }^{1, *}$, Yu Xie ${ }^{1}$, Runqiu Jiang ${ }^{1}$, Guoqiang Li $^{1}$, \\ Beicheng Sun ${ }^{1}$ \\ ${ }^{1}$ Liver Transplantation Center of the First Affiliated Hospital and Collaborative Innovation Center For Cancer Personalized \\ Medicine, Nanjing Medical University, Nanjing, Jiangsu Province, P.R. China \\ *These authors have contributed equally to this work
}

Correspondence to:

Beicheng Sun, e-mail: sunbc@njmu.edu.cn

Keywords: IncRNA, HCC, EPCAM, mTOR, prognosis

Received: April 27, $2015 \quad$ Accepted: October 16, 2015

Published: October 26, 2015

\section{ABSTRACT}

Hepatocellular carcinoma (HCC) is well known as the sixth most common malignant tumor and the third leading cause of cancer-related deaths globally. LINC00152 was documented as an important long non-coding RNA (IncRNA) involved in the pathogenesis of gastric cancer; however, the detailed mechanism of action of LINC00152 remains unknown. Here, based on the increased level of LINC00152 in HCC tissues, we found that LINC00152 could promote cell proliferation in vitro and tumor growth in vivo. Furthermore, microarray-based analysis indicated that LINC00152 could activate the mechanistic target of rapamycin(mTOR) pathway by binding to the promoter of EpCAM through a cis-regulation, as confirmed by Gal4- $\lambda N$ /BoxB reporter system. Thus, LINC00152 might be involved in the oncogenesis of HCC by activating the mTOR signaling pathway and might be a novel index for clinical diagnosis in the future.

\section{INTRODUCTION}

Every year, 500,000 people are affected by HCC, which is the sixth most common malignant tumor and the third leading cause of cancer-related deaths globally $[1,2]$. HCC contains a number of characteristics, including aggressiveness, invasiveness, especially intrahepatically, and frequent recurrence after resection [3]. Despite recent improvements in surgery and chemotherapy, HCC has a very high morbidity and mortality [4]. Therefore, the potential mechanisms, prognostic biomarkers and therapeutic targets of HCC require further investigation $[1,5,6]$.

The transcripts of greater than 200 nucleotides with no or little protein coding function, i.e. long noncoding RNAs, has attracted much attention in many fields $[7,8]$. Several lncRNAs were reported as biomarkers for predicting metastasis and diagnosis of multiple diseases, including hepatosis and HCC, as well as patient survival [9-12]. Recent studies have identified multiple functional effects of lncRNAs, including regulating gene expression through modulation of chromatin remodeling, controlling gene transcription, post-transcriptional mRNA processing, protein function or localization, and intercellular signaling [13-16]. The mechanisms of lncRNAs involved in liver disease have widely diverse functions, including DNA imprinting, $\mathrm{X}$ inactivation, DNA demethylation, gene transcription, and generation of other RNA molecules $[17,18]$. Furthermore, several researchers have discovered that lncRNAs could be modified epigenetically including by methylation, ubiquitination, and miRNA-induced regulation through a network [19].

The long noncoding RNA, LINC00152, was detected as differentially hypomethylated during hepatocarcinogenesis compared to healthy samples [20]. LINC00152 was reported to play an important role in cancer and has a 1.9-fold change of expression in gastric cancer [21]. The expression level of LINC00152 was increased significantly in both gastric carcinoma and gastric juice [22]. Meanwhile, LINC00152 can be detected in plasma, and one of the possible mechanisms of its stable existence in blood is that it is protected by exosomes [23]. LINC00152 appears to respond generally and highly to chemical stresses [24]. Besides, researcher has found LINC00152 may interact with THBS1 mediated by miR-18a-5p [25]. 
In this study, we explored whether LINC00152 is involved in hepatocarcinogenesis. Additionally, we attempted to identify the detailed effects and mechanisms of LINC00152 in HCC, both in vivo and in vitro.

\section{RESULTS}

\section{Hypomethylated LINC00152 was up-regulated in the primary $\mathrm{HCC}$ tissues and human $\mathrm{HCC}$ cell lines}

To investigate the LINC00152 expression level in HCC, we performed RT- PCR analysis on total RNA extracted from 102 pairs of HCC tissues. The expression level of LINC00152 in hepatocellular carcinoma was significantly increased, compared with matched normal tissue (Figure 1A). In addition, all HCC samples were divided into the LINC00152 low expression group $(n=51)$ and the high-expression group $(n=51)$, and the median was used as the cutoff value. The association between LINC00152 expression in $\mathrm{HCC}$ tissues and clinicopathological characteristics is presented in Table 1. We found no positive correlation with age, gender, HBV infection, cirrhosis, ALT, AFP, and vascular invasion. However, significant correlations with tumor size $(P=0.005)$ and Edmondson grade $(P=0.002)$ were obtained, indicating that LINC00152 might play a vital role in the development of HCC.

To investigate the subcellular location of LINC00152, the SurePrep ${ }^{\text {TM }}$ Nuclear/Cytoplasmic RNA Purification Kit was used. The transcript for LINC00152 was located mainly in the nucleus of MHCC-97H cells (Figure 1B). Further methylation analysis was performed by Bisulfite sequencing PCR (BSP). We found that the promoter region of LINC00152 was

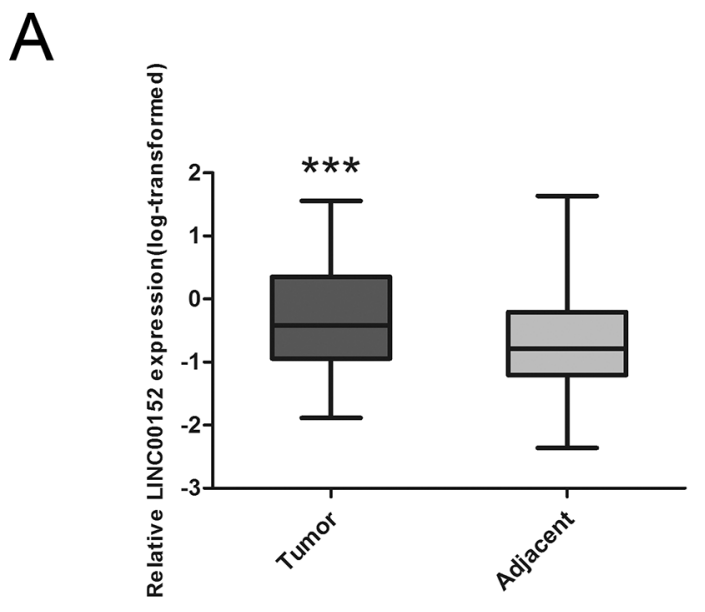

B

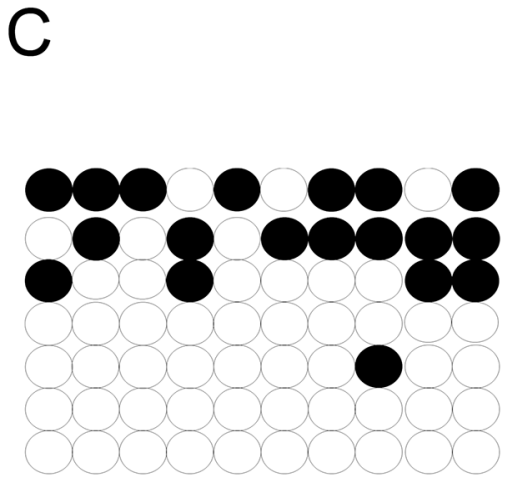

Tumor
Adjacent
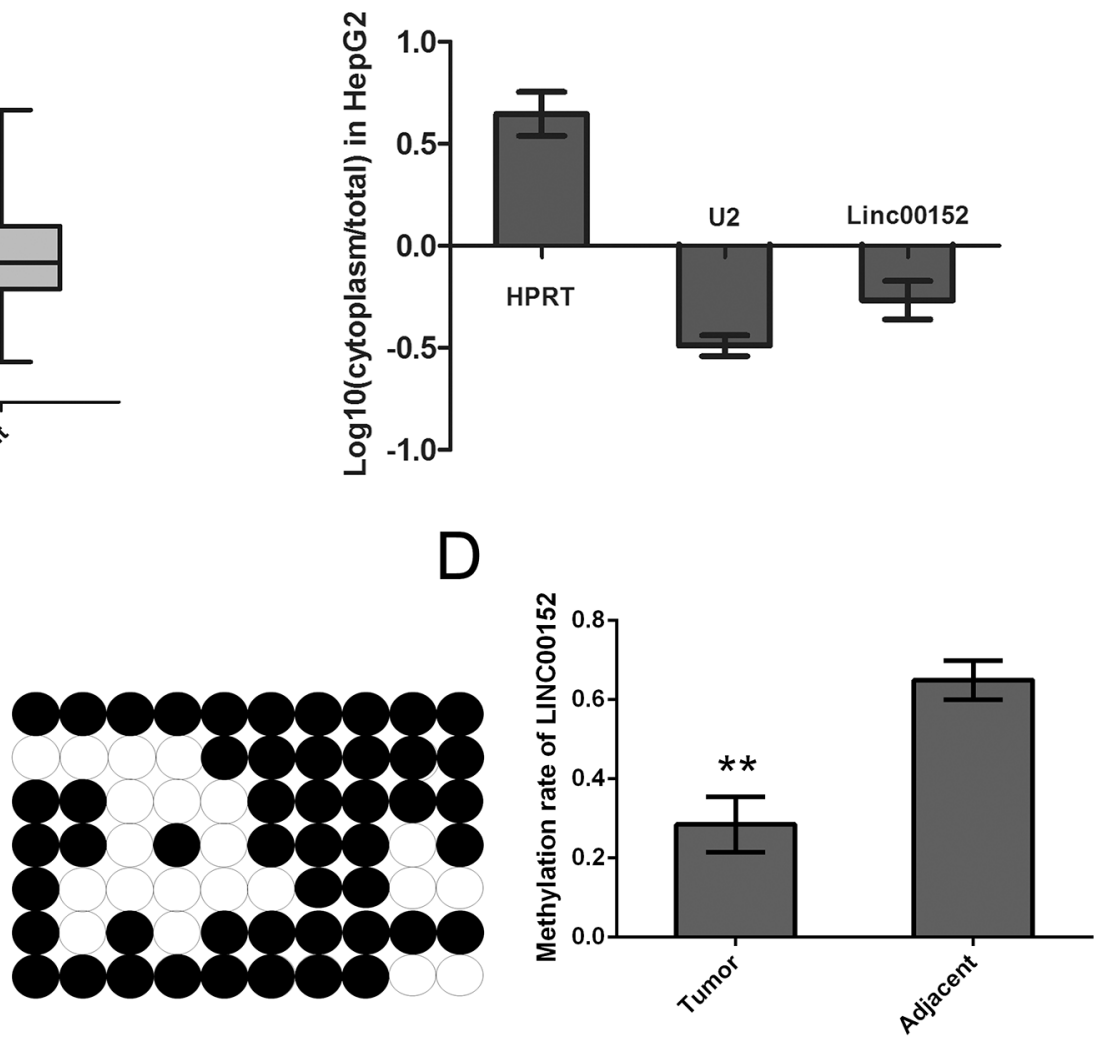

Figure 1: Aberrant up-regulated LINC00152 in HCC tissue samples and the subcellular location. A. An increased level of LINC00152 was detected in HCC tissues compared with the corresponding adjacent tissues $(n=102)$. All the expression of the LINC00152 normalized to $18 \mathrm{~S}$ using the $2^{-\Delta \Delta \mathrm{Ct}}$ method, then the expression of LINC00152 was transformed with $\log _{10}$. B. Subcellular $\operatorname{localization}$ investigation indicated that the transcript for LINC00152 was located mainly in the nucleus of MHCC-97H cell lines, according to the results of RT-PCR amplification with separated cytoplasmic RNA and nuclear RNA. HPRT was used as the control for cytoplasmic expression and U2 for cytonuclear expression. C, D. The bisulfite sequencing (BSP) method was used to detect methylation of CpG Island predicted in LINC00152. The methylation level of the LINC00152 promoter was downregulated in tumor tissues compared with the corresponding adjacent tissues. $(* *$ indicates $p<0.01$ while $* * *$ indicates $p<0.001)$. 
Table 1: Correlation between LINC00152 expression and clinicopathological characteristics of HCC patients $(n=102)$

\begin{tabular}{|c|c|c|c|c|}
\hline Characteristics & All Patients & $\begin{array}{l}\text { LINC00152 Low } \\
\text { Expression } \\
\left(<\text { Median }^{a}\right)\end{array}$ & $\begin{array}{l}\text { LINC00152 High } \\
\text { Expression } \\
\left(\geq \text { Median }^{\mathrm{a}}\right)\end{array}$ & $\begin{array}{c}p \\
\text { Chi-squared test } \\
p \text {-value }\end{array}$ \\
\hline No. & 102 & 51 & 51 & \\
\hline \multicolumn{5}{|l|}{ Age (years) } \\
\hline$<60$ & 77 & 35 & 42 & 0.107 \\
\hline$\geq 60$ & 25 & 16 & 9 & \\
\hline \multicolumn{5}{|l|}{ Gender } \\
\hline Male & 89 & 45 & 44 & 0.862 \\
\hline Female & 13 & 6 & 7 & \\
\hline \multicolumn{5}{|l|}{ HbeAg } \\
\hline Negative & 29 & 14 & 15 & 0.826 \\
\hline Positive & 73 & 37 & 36 & \\
\hline \multicolumn{5}{|l|}{ Cirrhosis } \\
\hline Absent & 16 & 7 & 9 & 0.586 \\
\hline Present & 86 & 44 & 42 & \\
\hline \multicolumn{5}{|l|}{ ALT (U/L) } \\
\hline$\leq 45$ & 59 & 29 & 30 & 0.841 \\
\hline$>45$ & 43 & 22 & 21 & \\
\hline \multicolumn{5}{|l|}{$\operatorname{AFP}(\mathrm{ng} / \mathrm{ml})$} \\
\hline$\leq 13.6$ & 23 & 10 & 13 & 0.477 \\
\hline$>13.6$ & 79 & 41 & 38 & \\
\hline \multicolumn{5}{|l|}{ Tumor size (cm) } \\
\hline$\leq 5$ & 42 & 28 & 14 & $0.005^{*}$ \\
\hline$>5$ & 60 & 23 & 37 & \\
\hline \multicolumn{5}{|l|}{ Vascular invasion } \\
\hline absent & 87 & 45 & 42 & 0.402 \\
\hline present & 15 & 6 & 9 & \\
\hline \multicolumn{5}{|c|}{ Edmondson grade } \\
\hline $\mathrm{I}+\mathrm{II}$ & 61 & 38 & 23 & $0.002 *$ \\
\hline $\mathrm{III}+\mathrm{IV}$ & 41 & 13 & 28 & \\
\hline
\end{tabular}

${ }^{a}$ The median expression level of Linc-152 was used as the cutoff

*Indicates $p$ value $<0.05$

hypomethylated in tumor tissues (Figure 1C, 1D). Since LINC00152 has not been reported in human HCC, we then started to analyze the second structure, non-coding function using bioinformatics software. As presented in Supplementary Figure S1A-S1C, LINC00152 was confirmed as the long non-coding RNA with a PhyloSCF score of -75.9943 .

\section{LINC00152 promotes cell proliferation in vitro}

To determine the functional role of LINC00152 in HCC, we first detected the expression of LINC00152 in the following six human HCC cell lines: HepG2, MHCC-97H, Huh7, SMMC-7721, Hep3B and SNU-423. As shown in Supplementary Figure S1D, LINC00152 expression 
levels were up-regulated in HCC cell lines compared to that in L02 normal human liver cells. Therefore, HepG2 and $\mathrm{MHCC}-97 \mathrm{H}$ were selected to investigate the effects of shRNA-mediated knockdown of LINC00152 on cell proliferation, apoptosis, invasion, migration and cell cycle.

Three LINC00152-specific shRNAs were evaluated for their knockdown efficiency, sh152-1 and sh152-3 were found to have higher silencing efficiency than sh152-2 (Supplementary Figure S1E). Thus, sh152-1 and sh152-3 were chosen for use in cell physiological function assays.

The data collected using the Cell Counting Kit-8 (CCK-8) and EdU (5-ethynyl-2'-deoxyuridine) cell proliferation assays suggested that down-regulation of LINC00152 inhibited the proliferation in both HepG2 and MHCC-97H cells (Figure 2A, 2B). Meanwhile, invasiveness, cell cycle and apoptosis analysis showed no significant difference between the sh152-treated cells and the normal control, in both of the cell lines mentioned above (Supplementary Figure S2A-S2C).

\section{LINC00152 induced a promotion of tumor growth in vivo}

To confirm the effects of LINC00152 on tumorigenesis in vivo, we conducted a nude mice xenograft experiment by subcutaneously injecting HepG2 and MHCC-97H cells whose LINC00152 was stably knocked down, or a mock vector (cells treated with a mock vector) into the flanks of approximately 4-6-week-old BALB/C nude mice. The left axilla was injected with mock cells, while the right side was injected with cells treated with sh152-1. We observed that tumor growth was significantly decreased compared with the controls (Figure 2C).

\section{The mTOR signal pathway was inhibited by the down-regulation of LINC00152 through a cis-regulation}

LINC00152 was reported to be up-regulated in multiple cancers and may play an important role in post-transcriptional regulation in cancer [22]. However, the specific signaling pathway involved in the abnormal expression of LINC00152 remained unknown. A microarray-based study was employed to demonstrate the potential signaling pathways. MHCC-97H cells were divided into two groups, including LINC00152 stable knockdown and the mock plasmid-treated cells. As presented in Figure 3A and 3B, genes with aberrant expression were selected with $4 / 0.25$ as the cut-off which were regarded as candidate genes for Gene Set Enrichment Analysis. Gene-annotation enrichment analysis indicated that the mTOR signal pathway was highly associated with LINC00152 down-regulation (Figure 3C).The anomalous activation of genes participating in the signaling pathway was confirmed by Western blot. As a result, the phosphorylation level of mTOR was decreased dramatically, along with the absence of LINC00152 in HepG2 and MHCC-97H cells (Figure 3D, 3E).

The HCC patients were divided into two groups (LINC00152 low and LINC00152 $2^{\text {high }}$ ) according to the median of LINC00152 expression in tumor tissues. We also analyzed the expression of mTOR and p-mTOR in the two groups described above. We found that the phosphorylation level of mTOR was decreased along with the lower expression of LINC00152 (Figure 4A). We next retrieved information from the National Center for Biotechnology Information (NCBI) Gene database (http://www.ncbi.nlm.nih.gov/gene) and found an mTOR-related gene, EpCAM, located near LINC00152 (Figure 4B). EpCAM is expressed in many human cancers with an epithelial origin. EpCAM has been implicated in cell invasion and may act as an oncogenic signaling protein. $\mathrm{EpCAM}(+) \mathrm{HCC}$ cells displayed hepatic cancer stem cell-like traits, including the abilities to self-renew and differentiate. Moreover, these cells were capable of initiating highly invasive HCC [26-29]. In our study, we further investigated the correlation between LINC00152 and EpCAM in clinical HCC tissues, and found that the expression levels of LINC00152 and EpCAM were positively correlated (Figure 4C). Then, we confirmed that the expression levels of LINC00152 and EpCAM were both decreased in MHCC-97L and SNU-423 cells when compared with HepG2 and MHCC-97H cells, using PCR and Western blot (Figure 4D). Interestingly, we found that silencing LINC00152 by sh152-1 resulted in the downregulation of EpCAM in HepG2 and MHCC-97H cells at both the mRNA and protein levels (Figure 4E). Then, we cloned the 5(-flanking region of EpCAM (-2000 bp region), regarded as the promoter region, into the pGL3Basic vector (pGL3-EpCAM). Dual luciferase reporter gene assay showed that the knockdown of LINC00152 could decrease the promoter activity of EpCAM in HepG2 and MHCC-97H cells (Figure 4F).

Gal4- $\lambda$ N/BoxB reporter system was employed as described previously to explore whether LINC00152 regulated EpCAM as a cis-pattern $[30,31]$. In this system, the BoxB RNA stem loop is fused to LINC00152; LUNAR1 was used as a positive control. The plasmid encoding a TK-luciferase gene under the control of five GAL4 UAS sites was co-transfected with plasmids encoding GAL4- $\lambda \mathrm{N}$ peptide as described above (Figure 5A). Ranilla luciferase was regarded as control in this system. The binding of Gal4$\lambda \mathrm{N}$ fusion was confirmed firstly (Figure 5B). Luciferase activity after co-transfection of the system indicated that tethering LINC00152 to this reporter gene could stimulate transcription of the reporter to a similar degree as LUNAR1 indicating that LINC00152 could function as a transcription activator (Figure 5C).

Further antisense oligonucleotides (ASO) targeting LINC00152 was designed, we found that the activation affection was significantly reduced comparing to control group (Figure 5D, 5E). 


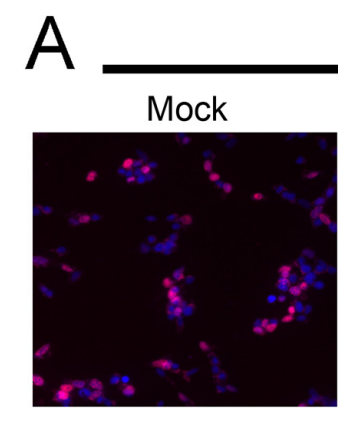

MHCC-97H
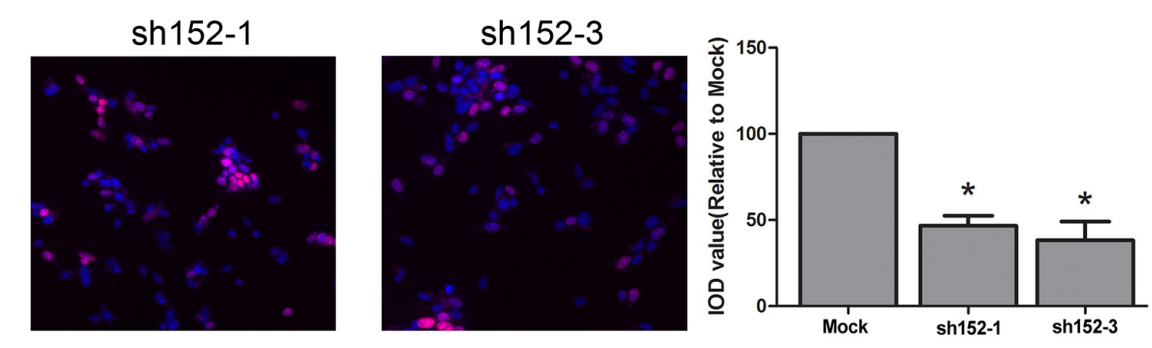

HepG2

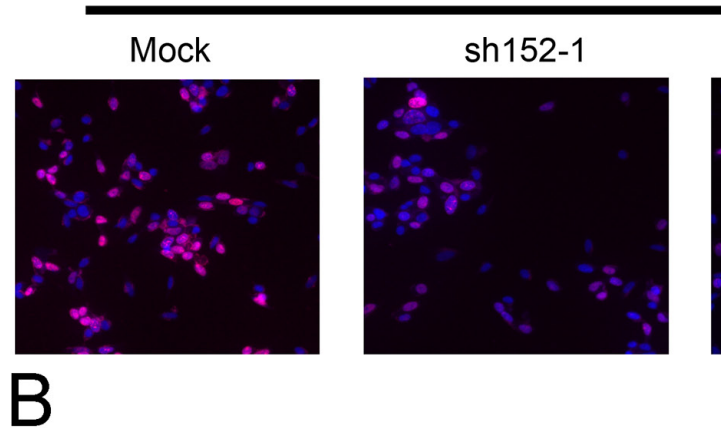

$\operatorname{sh} 152-3$
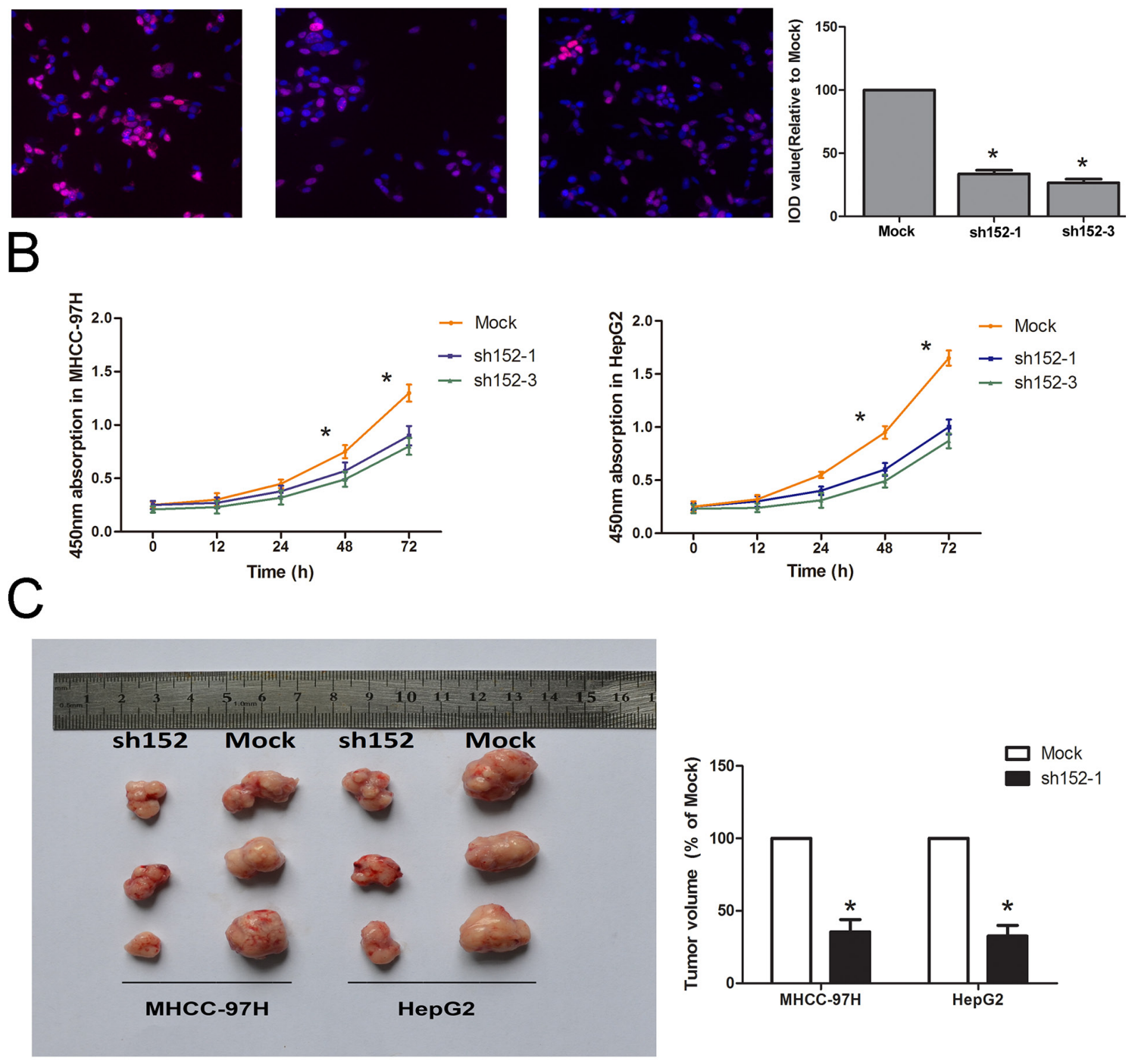

Figure 2: LINC00152 promoted cell proliferation in vitro and tumor growth in vivo. A. The EdU assay confirmed the functional role of LINC00152 in cell proliferation. Stable knockdown of LINC00152 reduced the proliferation of MHCC-97H and HepG2 after $24 \mathrm{~h}(P<0.01)$ compared with the negative control, with a magnification of 200 . The integral optical density value of cells treated with control plasmids was normalized to $100 \%$. B. The CCK-8 assay presented showed that a decreased level of LINC00152 inhibited the growth of MHCC-97H and HepG2. Absorbance at $450 \mathrm{~nm}$ is presented as the mean \pm SEM. C. All experiments were performed in triplicate and presented as the mean \pm SEM. * indicates a significant difference compared with the control group $(P<0.05)$. C: Bilateral axillae of BALB/C nude mice were transplanted subcutaneously with MHCC-97H or HepG2 cells stably expressing LINC00152 shRNA or the mock $(n=5)$. The volume of each tumor was calculated as the length $\times$ width $^{2} \times 0.5$. The tumor volume of cells treated with controls was normalized to $100 \%$. Data are presented as the mean \pm SEM. * indicates a significant difference compared with controls $(P<0.05)$. 


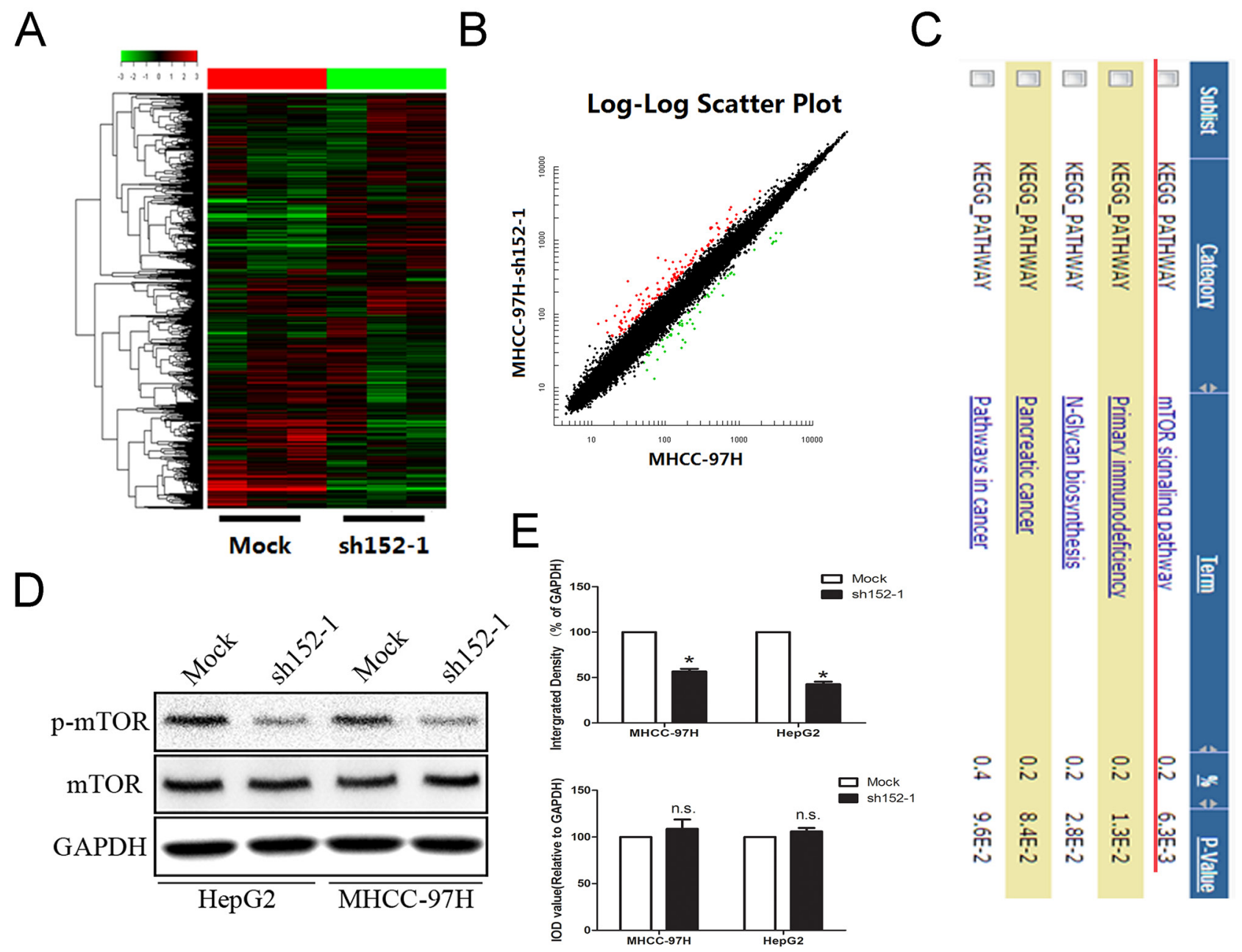

Figure 3: Microarray investigation indicated that the mTOR signal pathway was suppressed by the absence of LINC00152. A, B. Heatmap analysis and scatter plot results present different gene expression with the cutoff value set at $4 / 0.25$. C. Gene annotation for enrichment of the candidate genes. D. Western blot was applied to confirm that the aberrant suspension of the mTOR signaling pathway was induced by the loss of LINC00152. GAPDH was used as a loading control. E. Integral optical density (IOD) was calculated for each band. Data are presented as the mean \pm SEM. * indicates $P<0.05$.

\section{DISCUSSION}

HCC is a worldwide disease with a high incidence. Despite advanced therapeutic treatments such as surgery, chemotherapy and radiotherapy, more than 600,000 people die of HCC each year [32]. Hence, the exploration of the pathogenesis and development of effective treatments is essential. A number of LncRNAs have been discovered to play a vital role in hepatocarcinogenesis and HCC progression [33], including, HULC [34], HOTTIP [14], HOTAIR [35], MVIH [36]. It has been documented that the expression of LINC00152 in gastric cancer is higher compared to normal gastric tissue [21].

Along the digestive tract, we detected abnormal expression of LINC00152 in HCC, which was significantly correlated with tumor size. We then confirmed the proliferative promotion of LINC00152 in vitro and in vivo. To further investigate the potential signaling pathway and target gene regulated by LINC00152, we used microarray and bioinformatics analyses. Based on the data of the transcriptome microarray assay, signaling pathway enrichment and target gene differential analysis, we discovered that LINC00152 potentially regulates the mTOR signaling pathway, and confirmed this with Western bolting. We therefore used the dual luciferase reporter gene assay to explore how LINC00152 targets EpCAM at the transcriptional level. We found that the knockdown of LINC00152 could decrease the promoter activity of EpCAM in HepG2 and MHCC-97H cells. Combined with other research, we found that the direct interaction between IncRNAs and the promoter of target genes is one of the major regulatory paradigms of lncRNAs.

Cis-acting lncRNAs whose regulatory functions are restricted to genes on the same chromosome are likely to operate only at the level of transcription [37]. Taro et al. identified two TCF binding elements (TBE1; -TTCAAAG-, 


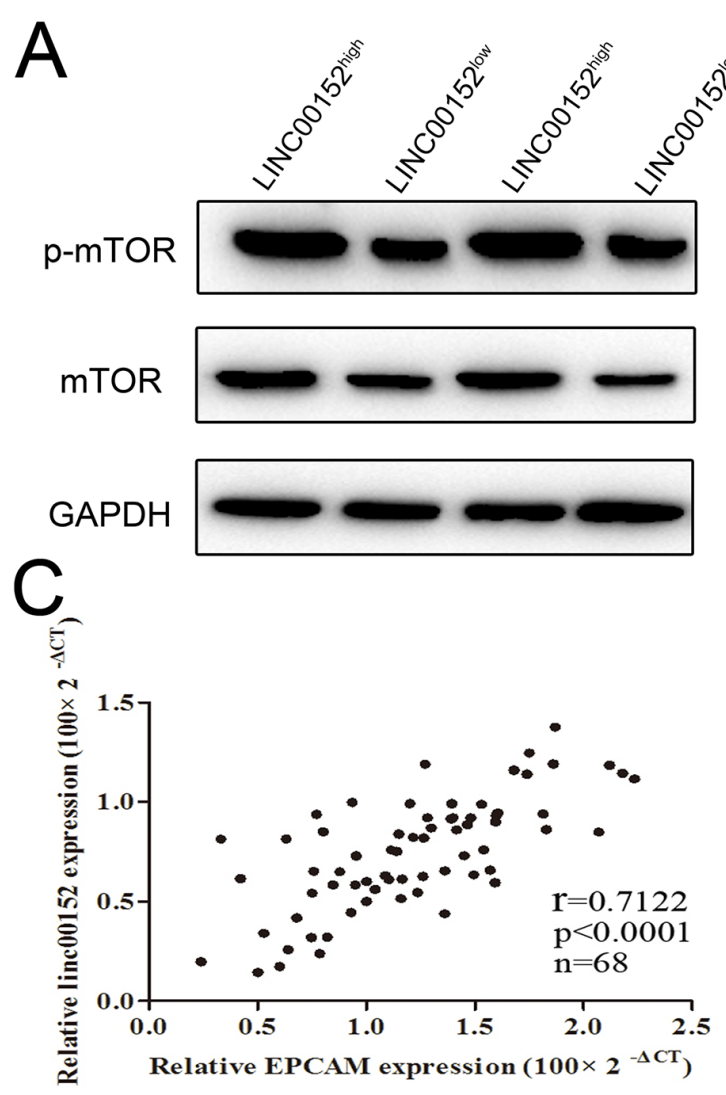

EPCAM
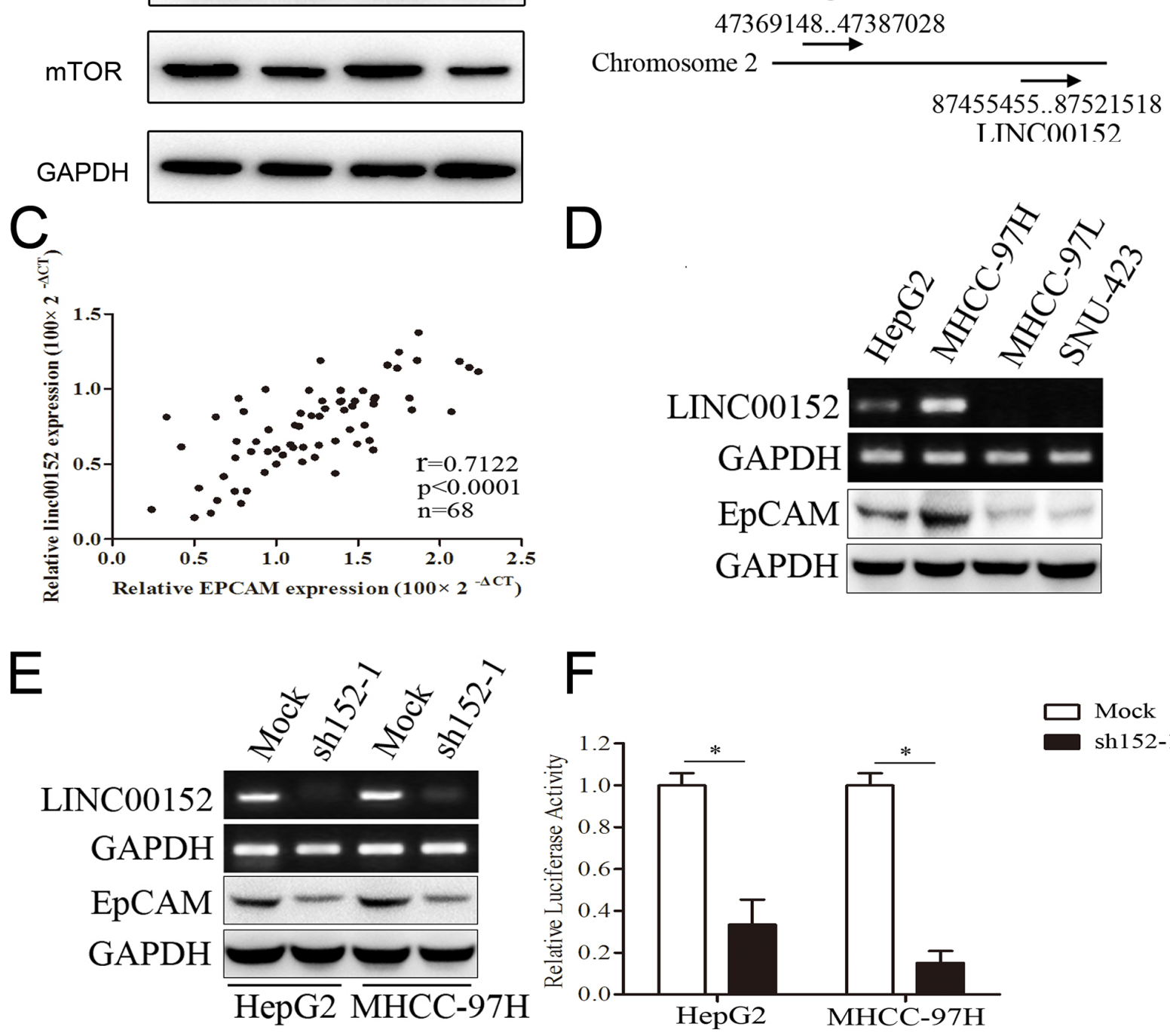

Figure 4: LINC00152 activated mTOR via binding the promoter of EpCAM. A. Different expression of mTOR and $p$-mTOR in human tissues in LINC00152 ${ }^{\text {low }}$ and LINC00152 ${ }^{\text {high }}$ groups. B. Location of LINC00152 and EpCAM in Chromosome 2. C. Correlation expression levels of LINC00152 and EpCAM using Pearson analysis. D. Different RNA expression levels of LINC00152 and EpCAM in cell lines. E. RNA expression level of LINC00152 in cells treated with sh152-1 and control (top panel). Protein expression level of EpCAM in cells treated with sh152-1 and control (lower panel). F. Cells were co-transfected with the promoter region of EpCAM with reporter, the Renilla luciferase vector pRL-SV40 and LINC00152 shRNA, or control for $48 \mathrm{~h}$. Both firefly and Renilla luciferase activities were measured in the same sample. Firefly luciferase signals were normalized with Renilla luciferase signals. Cells treated with controls of miRNA were normalized to $100 \%$.

TBE2; -CTTTGAT-) in the EpCAM promoter that specifically bind to the TCF/ $\beta$-catenin complex. We found two sequences (432-438; -CAUUGAA-, 609-616; -GAUCACAG-) in the LINC00152 that may bind to TBE1 and TBE2 [29]. In human distal cells, the lncRNA HOTTIP recruits the histone H3K4-modifying complex MLL1 by binding to WDR5, targeting this complex to the HOXA locus.
Thus, chromatin modifications together with higherorder chromosomal looping bring the HOTTIP RNA in close proximity to HOXA genes $[38,39]$. In our study, the LINC00152 may be required for maintaining transcriptionally active EpCAM genes at the $5 \square$ end of the cluster.

In conclusion, our findings demonstrate the upregulation of LINC00152 in human HCC,. Aberrant 

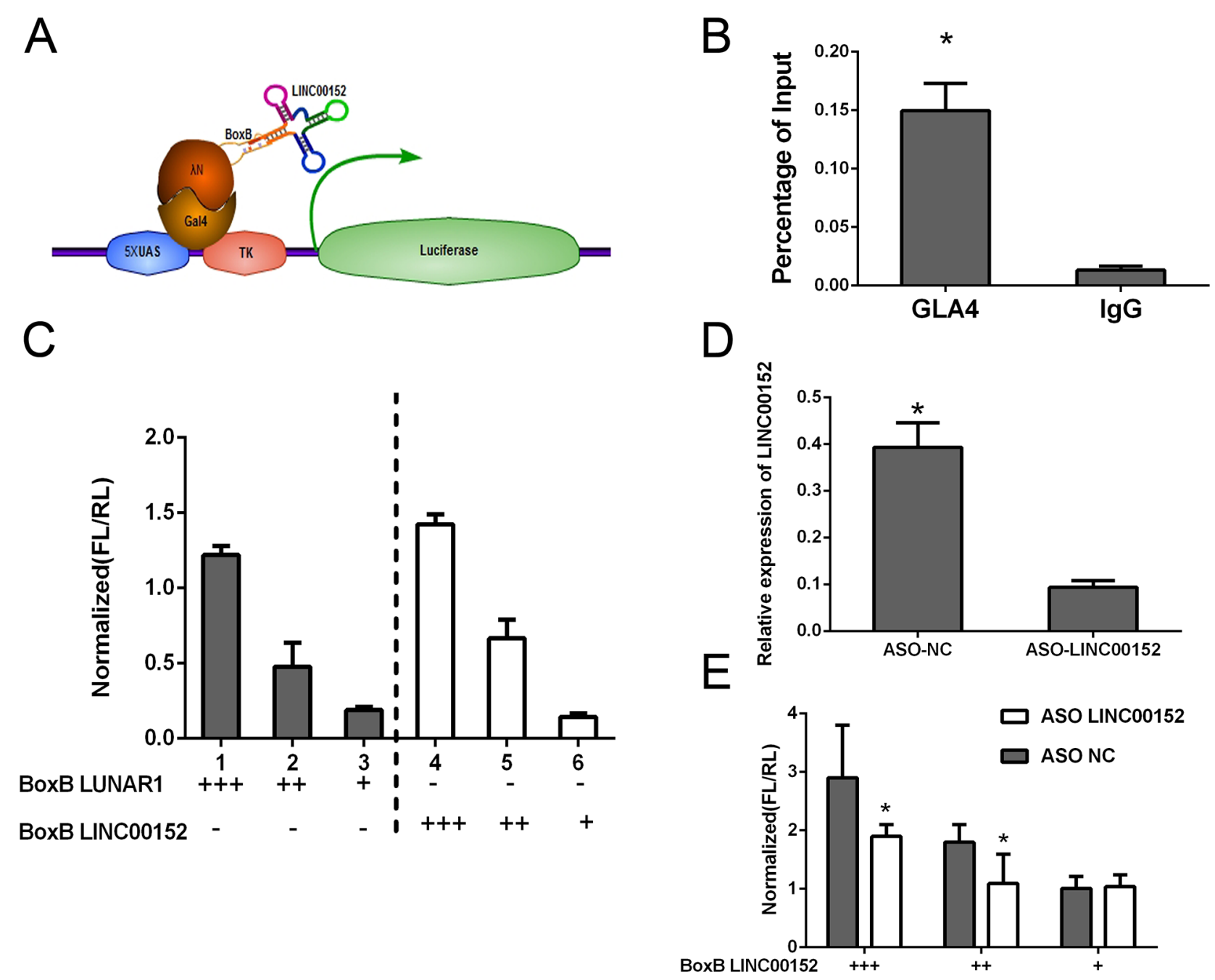

Figure 5: Cis-regulation of LINC00152. A. Schematic figure for Gal4- $\lambda \mathrm{N} / \mathrm{BoxB}$ reporter system. B. The binding of Gal4- $\lambda \mathrm{N}$ fusion was confirmed by ChIP. C. Luciferase reporter activity in experiments where BoxB-tagged LINC00152 (right) or LUNAR1 (left) were cotransfected with Gal4-IN. D. qPCR following ASO knockdown of LINC00152 in luciferase assay. E. Reporter assay showing relative reporter gene activity when BoxB-LINC00152 was cotransfected with either control or ASO targeting LINC00152. Data was normalized to Ranilla luciferase. Data presented as the mean \pm SEM. * indicates $p$ value $<0.05$.

expression of LINC00152 increases EpCAM levels, resulting in the activation of the mTOR signaling pathway, and afterwards, causing the proliferation of HCC both in vitro and in vivo. These findings contribute to a better understand of the mechanism of action of IncRNA. Our data indicate that LINC00152 might be involved in HCC development, which may play a vital role in the tumorigenesis of HCC, and may serve as potential target for therapy in the future.

\section{MATERIALS AND METHODS}

\section{Clinical samples and cell cultures}

The clinical data were obtained from 102 cases of patients who underwent liver cancer radical resection surgeryduring January 2010 to December 2012 at The
First Affiliated Hospital of Nanjing Medical University (Nanjing, China). The specimens were stored at $-80^{\circ} \mathrm{C}$ immediately after surgery. No patient had a history of exposure to either radiotherapy or chemotherapy before the surgery, and no other co-occurring cancers were diagnosed. This study was approved by the Ethical Committee of the First Affiliated Hospital of Nanjing Medical University, and every patient provided written informed consent.

The human HCC cell lines used in this study were obtained from the American Type Culture Collection (Manassas, VA, USA), and included HepG2, Hep3B and SNU-423 cells. Huh7, L02, SMMC-7721 and MHCC97H cells were purchased from the Cellbank of the Chinese Academy of Sciences in Shanghai. All of the cell lines were maintained in an atmosphere of $5 \% \mathrm{CO}_{2}$ and grown in DMEM medium (Thermo, Beijing, China) 
supplemented with $10 \%$ fetal bovine serum (Thermo). Cell line authentication was performed by short tandem repeat (STR) profiling before the initiation of this study.

\section{Quantitative real time polymerase chain reaction (qRT-PCR)}

Quantitative real time polymerase chain reaction (qRT-PCR) was performed to determine the expression levels of LINC00152 and other related mRNAs. Total RNAs were extracted from frozen tissues and cell lines using TRIzol reagent, as described by the manufacturer's protocol (Invitrogen, CA, USA). One microgram of total RNA from all samples was reverse transcribed using the PrimeScript RT Master Mix kit (Takara, Tokyo, Japan). Beta-actin was used as an internal control. All the primer sequences are shown in Supplementary Table S1. qRT-PCR was performed using an ABI Prism 7900HT instrument (Applied Biosystems, CA, USA), according to the instructions of the manufacturer. The gene expression data were analyzed using the $2^{-\Delta \Delta \mathrm{Ct}}$ method.

\section{Gene silencing of LINC00152 in HCC cell lines}

To knock down LINC00152 expression, an shRNA sequence targeting LINC00152 was cloned into the lentivirus vector pLL3.7, and designated as pLL3.7sh152-1 and pLL3.7-sh152-2. The vectors also included the gene for green fluorescent protein (GFP). The sequences are presented in Supplementary Table S1. The hairpin shRNA with no sequence homology to human genes provided by the same manufacturer was used as the negative control, named the mock group. All constructs were sequence-verified. Details are available on request. Recombinant lentivirus was generated from 293T cells by cotransfection of pdelta-8.91, pVSVG together with pLL3.7-sh152-1 and pLL3.7-sh152-2. The GFPpositive cells were sorted 3 days after infection with the lentiviruses. The stable cells infected with LINC00152 shRNA were selected by puromycin. After cells treated with lentivirus for $48 \mathrm{~h}$, medium containing puromycin was added and the medium was replaced every 2 days.A pure population of infected cells was sorted based on GFPexpression by flow cytometry; more than $98 \%$ of the cells were GFP-positive after sorting. A total of $1.5 \times 10^{5}$ cells were seeded in $35 \mathrm{~mm}^{2}$ tissue culture dishes for $24 \mathrm{~h}$. The knock-down efficiency of the LINC00152 by shRNAs was monitored by qRT-PCR. The cells were then subjected to RNA extraction or functional assays.

\section{Cell proliferation assay}

Cell proliferation was assayed using CCK-8(Dojin Laboratories, Kumamoto, Japan) and EdU(Millipore, MA, America), according to the manufacturer's instructions. The mock and infected cells were seeded at a density of $1 \times 10^{4}$ cells/well in 96-well flat-bottom plates, and cultured for the CCK-8 and EdU assays.

After a period of time $(0,12,24,48,72 \mathrm{~h})$ following the seeding of the cells, CCK- 8 was added to each well containing $100 \mu \mathrm{L}$ of the culture medium, and the plate was incubated for $3 \mathrm{~h}$ at 37(C. Viable cells were evaluated by measuring the absorbance at $450 \mathrm{~nm}$, using a microplate reader. The EdU assay was performed after the cells were cultured for 48 h. Finally, the cells were washed, mounted with mounting medium containing DAPI (Santa Cruz Biotechnology, CA, USA), and imaged using an Olympus FV1000 confocal microscope (Olympus, NY, USA).

\section{Cell cycle and apoptosis analysis}

Cells were infected with sh152-1, as well as with normal controls, and were harvested $48 \mathrm{~h}$ later. For the cell cycle assay, cells were fixed in $75 \%$ alcohol, and stained with propidium iodide (Sigma, MO, USA). For the analysis of apoptosis, cells were washed in PBS, and then mixed with the Annexin V-FITC Apoptosis Detection Kit (BD Biopharmingen, NJ, USA) for $15 \mathrm{~min}$ in the dark. All experiments were analyzed using the BD Biosciences FACSCalibur Flow Cytometer (BD Biasciences, NJ, USA). The tests were repeated three times, and triplicate wells were used for each condition.

\section{The subcutaneous xenotransplantation model}

Animal care and euthanasia were approved by the Nanjing Medical University Animal Studies Committee. HepG2 and MHCC-97H cells and control cells $\left(1 \times 10^{7}\right)$ stably knocked down for LINC00152 expression were implanted subcutaneously into the bilateral axillae of twelve BALB/C nude mice. Tumors were measured every week after implantation, and the volume of each tumor was calculated (length $\times$ width $^{2} \times 0.5$ ). All mice were sacrificed 5 weeks afterwards, and the xenografts were peeled off subcutaneously. The weights of the xenografts in each group were compared.

\section{Protein extraction and Western blotting}

Total protein was extracted from tissues or cultured cells using RIPA buffer containing phenylmethanesulfonylfluoride (PMSF) (Beyotime, Nantong, China). Protein samples were loaded equally in each lane, then resolved using SDS-PAGE (Beyotime, Nantong, China), and transferred onto a nitrocellulose membrane. The membranes were blocked with $5 \%$ BSA for $1 \mathrm{~h}$ at room temperature and incubated at $4{ }^{\circ} \mathrm{C}$ overnight with primary antibodies purchased from Abcam (London, UK). Sequentially, the secondary antibodies were conjugated to horseradish peroxidase, and the proteins were visualized via chemiluminescence (Millipore, CA, USA). GAPDH (Abcam, London, UK) was used to normalize the quantity 
of the protein. The integrated density of the band was quantified using Image Lab software (Bio-Rad, CA, USA).

\section{Microarray assay and bioinformatics analyses}

Total RNA was extracted from HepG2 and MHCC-97H cells in which LINC00152 was stably knocked down, and control cells were treated with the corresponding empty plasmid, PLL3.7, and was amplified and transcribed into fluorescent cDNA. Labeled samples were hybridized to the Human Roche NimbleGen mRNA microarray (Roche, CA, USA). Bioinformatics analyses were conducted using the MAS3.0 system (CapitalBio, Beijing, China) and DAVID Functional Annotation Bioinformatics Microarray Analysis (http://david.abcc. ncifcrf.gov/).

\section{Gal4- $\lambda$ N/BoxB reporter assay}

In this system, the BoxB RNA stem loop is fused to LINC00152, LUNAR1 was used as a positive control as described previously [30]. The plasmid encoding a TK-luciferase gene under the control of five GAL4 UAS sites was co-transfected with plasmids encoding GAL4- $\lambda N$ peptide fused to a C-terminal GFP tag, BoxB as described above. Ranilla luciferase was regarded as control in this system. The binding of Gal4- $\lambda \mathrm{N}$ fusion was confirmed firstly.

\section{ASO technology}

Antisense oligonucleotides (ASOs) were designed using the IDT Antisense Design Tool (http:// www.idtdna.com) using the chimeric 25-mer setting. The top 3 ASOs generated by the design tool were ordered and tested for knockdown efficiency for further investigation. Sequence for the control group in ASO assay was taken from Thomas Trimarchi, et al [30]. For ASO knockdown in BoxB tethering experiments, ASOs were co-transfected with plasmid DNA at $50 \mathrm{nM}$.

\section{Dual luciferase reporter gene assay}

The EpCAM promoter sequence $(-2000 \mathrm{bp})$ was cloned into the plasmid pGL3-Basic. The treated cells harvested $48 \mathrm{~h}$ after siRNA treatment, and the firefly luciferase expression was measured and normalized to Renilla activities. Dual-luciferase assays (Promega, Madison, WI) were performed according to the manufacturer's protocol, and detected with a Fluoroskan microplate reader (Thermo Labsystems, Helsinki, Finland).

\section{Statistical analysis}

All experiments were performed in triplicate, and repeated at least three times. Data were expressed as mean \pm SD. Differences between two independent groups were tested with Student's $t$-test. All statistical analyses were carried out using SPSS version 18.0 and presented with Graphpad prism software.The results were considered to be statistically significant at $P<0.05$.

\section{ACKNOWLEDGMENTS AND FUNDING}

This work was supported by grants from the National Natural Science Foundation for Distinguished Young Scholars (81225017 to B.S.); The State Key Program of National Natural Science of China $(81430062$ to B.S.); National Basic Research Program of China (2012CB910800 to B.S.), The National Natural Science Foundation (81201528 to R.J.; 81201880 to L.D.), This work also supported in part by the program for Development of Innovative Research Team in the First Affiliated Hospital of NJMU, and the Priority Academic Program of Jiangsu Higher Education Institutions. B.S. is Yangtze River scholars Distinguished Professor.

\section{CONFLICTS OF INTEREST}

The authors declare no competing financial interests.

\section{REFERENCES}

1. Block TM, Mehta AS, Fimmel CJ, Jordan R. Molecular viral oncology of hepatocellular carcinoma. Oncogene. 2003; 22:5093-5107.

2. Shen Q, Fan J, Yang XR, Tan Y, Zhao W, Xu Y, Wang N, Niu Y, Wu Z, Zhou J, Qiu SJ, Shi YH, Yu B, Tang N, Chu W, Wang M, et al. Serum DKK1 as a protein biomarker for the diagnosis of hepatocellular carcinoma: a large-scale, multicentre study. The Lancet Oncology. 2012; 13:817-826.

3. Sahasrabuddhe VV, Gunja MZ, Graubard BI, Trabert B, Schwartz LM, Park Y, Hollenbeck AR, Freedman ND, McGlynn KA. Nonsteroidal anti-inflammatory drug use, chronic liver disease, and hepatocellular carcinoma. J Natl Cancer Inst. 2012; 104:1808-1814.

4. Di Bisceglie AM. Issues in screening and surveillance for hepatocellular carcinoma. Gastroenterology. 2004; 127:S104-107.

5. El-Serag HB. Hepatocellular carcinoma: recent trends in the United States. Gastroenterology. 2004; 127:S27-34.

6. El-Serag HB, Rudolph KL. Hepatocellular carcinoma: epidemiology and molecular carcinogenesis. Gastroenterology. 2007; 132:2557-2576.

7. Spizzo R, Almeida MI, Colombatti A, Calin GA. Long noncoding RNAs and cancer: a new frontier of translational research? Oncogene. 2012; 31:4577-4587.

8. Tang J, Jiang R, Deng L, Zhang X, Wang K, Sun B. Circulation long non-coding RNAs act as biomarkers for 
predicting tumorigenesis and metastasis in hepatocellular carcinoma. Oncotarget. 2015; 6:4505-4515.

9. Cesana M, Cacchiarelli D, Legnini I, Santini T, Sthandier O, Chinappi M, Tramontano A, Bozzoni I. A long noncoding RNA controls muscle differentiation by functioning as a competing endogenous RNA. Cell. 2011; 147:358-369.

10. Batista PJ, Chang HY. Long noncoding RNAs: cellular address codes in development and disease. Cell. 2013; 152:1298-1307.

11. Xu D, Yang F, Yuan JH, Zhang L, Bi HS, Zhou CC, Liu F, Wang F, Sun SH. Long noncoding RNAs associated with liver regeneration 1 accelerates hepatocyte proliferation during liver regeneration by activating Wnt/beta-catenin signaling. Hepatology. 2013; 58:739-751.

12. Takahashi K, Yan I, Haga H, Patel T. Long noncoding RNA in liver diseases. Hepatology. 2014; 60:744-753.

13. Wilusz JE, Sunwoo H, Spector DL. Long noncoding RNAs: functional surprises from the RNA world. Genes Dev. 2009; 23:1494-1504.

14. Quagliata L, Matter MS, Piscuoglio S, Arabi L, Ruiz C, Procino A, Kovac M, Moretti F, Makowska Z, Boldanova T, Andersen JB, Hammerle M, Tornillo L, Heim MH, Diederichs S, Cillo C, et al. Long noncoding RNA HOTTIP/ HOXA13 expression is associated with disease progression and predicts outcome in hepatocellular carcinoma patients. Hepatology. 2014; 59:911-923.

15. Hammerle M, Gutschner T, Uckelmann H, Ozgur S, Fiskin E, Gross M, Skawran B, Geffers R, Longerich T, Breuhahn K, Schirmacher P, Stoecklin G, Diederichs S. Posttranscriptional destabilization of the liver-specific long noncoding RNA HULC by the IGF2 mRNA-binding protein 1 (IGF2BP1). Hepatology. 2013; 58:1703-1712.

16. Tang J, Zhuo H, Zhang X, Jiang R, Ji J, Deng L, Qian X, Zhang F, Sun B. A novel biomarker Linc00974 interacting with KRT19 promotes proliferation and metastasis in hepatocellular carcinoma. Cell Death Dis. 2014; 5:e1549.

17. Tuck AC, Tollervey D. A transcriptome-wide atlas of RNP composition reveals diverse classes of mRNAs and lncRNAs. Cell. 2013; 154:996-1009.

18. Ulitsky I, Bartel DP. lincRNAs: genomics, evolution, and mechanisms. Cell. 2013; 154:26-46.

19. Necsulea A, Soumillon M, Warnefors M, Liechti A, Daish T, Zeller U, Baker JC, Grutzner F, Kaessmann H. The evolution of lncRNA repertoires and expression patterns in tetrapods. Nature. 2014; 505:635-640.

20. Neumann O, Kesselmeier M, Geffers R, Pellegrino R, Radlwimmer B, Hoffmann K, Ehemann V, Schemmer P, Schirmacher P, Lorenzo Bermejo J, Longerich T. Methylome analysis and integrative profiling of human HCCs identify novel protumorigenic factors. Hepatology. 2012; 56:1817-1827.

21. Cao WJ, Wu HL, He BS, Zhang YS, Zhang ZY. Analysis of long non-coding RNA expression profiles in gastric cancer. World journal of gastroenterology : WJG. 2013; 19:3658-3664.
22. Pang Q, Ge J, Shao Y, Sun W, Song H, Xia T, Xiao B, Guo J. Increased expression of long intergenic non-coding RNA LINC00152 in gastric cancer and its clinical significance. Tumour biology : the journal of the International Society for Oncodevelopmental Biology and Medicine. 2014; 35:5441-5447.

23. Li Q, Shao Y, Zhang X, Zheng T, Miao M, Qin L, Wang B, Ye G, Xiao B, Guo J. Plasma long noncoding RNA protected by exosomes as a potential stable biomarker for gastric cancer. Tumour biology : the journal of the International Society for Oncodevelopmental Biology and Medicine. 2015; 36:2007-2012.

24. Tani H, Onuma Y, Ito Y, Torimura M. Long non-coding RNAs as surrogate indicators for chemical stress responses in human-induced pluripotent stem cells. PloS one. 2014; 9:e106282.

25. Xia T, Liao Q, Jiang X, Shao Y, Xiao B, Xi Y, Guo J. Long noncoding RNA associated-competing endogenous RNAs in gastric cancer. Sci Rep. 2014; 4:6088.

26. Kimura O, Takahashi T, Ishii N, Inoue Y, Ueno Y, Kogure T, Fukushima K, Shiina M, Yamagiwa Y, Kondo Y, Inoue J, Kakazu E, Iwasaki T, Kawagishi N, Shimosegawa T, Sugamura K. Characterization of the epithelial cell adhesion molecule (EpCAM)+ cell population in hepatocellular carcinoma cell lines. Cancer science. 2010; 101:2145-2155.

27. Yamashita T, Budhu A, Forgues M, Wang XW. Activation of hepatic stem cell marker EpCAM by Wnt-beta-catenin signaling in hepatocellular carcinoma. Cancer research. 2007; 67:10831-10839.

28. Yamashita T, Forgues M, Wang W, Kim JW, Ye Q, Jia H, Budhu A, Zanetti KA, Chen Y, Qin LX, Tang ZY, Wang XW. EpCAM and -Fetoprotein Expression Defines Novel Prognostic Subtypes of Hepatocellular Carcinoma. Cancer research. 2008; 68:1451-1461.

29. Yamashita T, Ji J, Budhu A, Forgues M, Yang W, Wang HY, Jia H, Ye Q, Qin LX, Wauthier E, Reid LM, Minato H, Honda M, Kaneko S, Tang ZY, Wang XW. EpCAM-positive hepatocellular carcinoma cells are tumor-initiating cells with stem/progenitor cell features. Gastroenterology. 2009; 136:1012-1024.

30. Trimarchi T, Bilal E, Ntziachristos P, Fabbri G, DallaFavera R, Tsirigos A, Aifantis I. Genome-wide mapping and characterization of Notch-regulated long noncoding RNAs in acute leukemia. Cell. 2014; 158:593-606.

31. Wang Y, Wu P, Lin R, Rong L, Xue Y, Fang Y. LncRNA NALT interaction with NOTCH1 promoted cell proliferation in pediatric $\mathrm{T}$ cell acute lymphoblastic leukemia. Sci Rep. 2015; 5:13749.

32. Ferenci P, Fried M, Labrecque D, Bruix J, Sherman M, Omata M, Heathcote J, Piratsivuth T, Kew M, Otegbayo JA, Zheng SS, Sarin S, Hamid S, Modawi SB, Fleig W, Fedail S, et al. World Gastroenterology Organisation Guideline. Hepatocellular carcinoma (HCC): a global perspective. Journal of gastrointestinal and liver diseases : JGLD. 2010; 19:311-317. 
33. Gibb EA, Brown CJ, Lam WL. The functional role of long non-coding RNA in human carcinomas. Molecular cancer. 2011; 10:38.

34. Liu Y, Pan S, Liu L, Zhai X, Liu J, Wen J, Zhang Y, Chen J, Shen $\mathrm{H}, \mathrm{Hu} \mathrm{Z}$. A genetic variant in long non-coding RNA HULC contributes to risk of HBV-related hepatocellular carcinoma in a Chinese population. PloS one. 2012; 7:e35145.

35. Yang Z, Zhou L, Wu LM, Lai MC, Xie HY, Zhang F, Zheng SS. Overexpression of long non-coding RNA HOTAIR predicts tumor recurrence in hepatocellular carcinoma patients following liver transplantation. Annals of surgical oncology. 2011; 18:1243-1250.

36. Yuan SX, Yang F, Yang Y, Tao QF, Zhang J, Huang G, Wang RY, Yang S, Huo XS, Zhang L, Wang F, Sun SH, Zhou WP. Long noncoding RNA associated with microvascular invasion in hepatocellular carcinoma promotes angiogenesis and serves as a predictor for hepatocellular carcinoma patients' poor recurrence-free survival after hepatectomy. Hepatology. 2012; 56:2231-2241.

37. Guenzl PM, Barlow DP. Macro lncRNAs: a new layer of cis-regulatory information in the mammalian genome. RNA biology. 2012; 9:731-741.

38. Wang KC, Yang YW, Liu B, Sanyal A, CorcesZimmerman R, Chen Y, Lajoie BR, Protacio A, Flynn RA, Gupta RA, Wysocka J, Lei M, Dekker J, Helms JA, Chang HY. A long noncoding RNA maintains active chromatin to coordinate homeotic gene expression. Nature. 2011; 472:120-124.

39. Guil S, Esteller M. Cis-acting noncoding RNAs: friends and foes. Nature structural \& molecular biology. 2012; 19:1068-1075. 